\title{
Fitness outcomes from a randomised controlled trial of exercise training for men with prostate cancer: the ENGAGE study
}

\author{
Cadeyrn J. Gaskin ${ }^{1} \cdot$ Steve F. Fraser ${ }^{2}$ Patrick J. Owen ${ }^{2} \cdot$ Melinda Craike ${ }^{1,3}$. \\ Liliana Orellana $^{4}$ • Patricia M. Livingston ${ }^{1}$
}

Received: 11 November 2015 / Accepted: 9 April 2016 /Published online: 20 April 2016

(C) The Author(s) 2016. This article is published with open access at Springerlink.com

\begin{abstract}
Purpose The main purpose of this study was to investigate the effects of a 12-week, clinician-referred, community-based exercise training program with supervised and unsupervised sessions for men with prostate cancer. The secondary purpose was to determine whether androgen deprivation therapy (ADT) modified responses to exercise training.

Methods Secondary analysis was undertaken on data from a multicentre cluster randomised controlled trial in which 15 clinicians were randomly assigned to refer eligible patients to an exercise training intervention $(n=8)$ or to provide usual care $(n=7)$. Data from 119 patients (intervention $n=53$, control $n=66$ ) were available for this analysis. Outcome measures included fitness and physical function, anthropometrics, resting heart rate, and blood pressure.

Results Compared to the control condition, men in the intervention significantly improved their 6-min walk distance $\left(M_{\text {diff }}=49.98 \mathrm{~m}, p_{\text {adj }}=0.001\right)$, leg strength $\left(M_{\text {diff }}=21.82 \mathrm{~kg}, p\right.$ adj $=0.001)$, chest strength $\left(M_{\text {diff }}=6.91 \mathrm{~kg}, p_{\text {adj }}=0.001\right), 30-\mathrm{s}$ sit-to-stand result $\left(M_{\text {diff }}=3.38\right.$ reps, $\left.p_{\text {adj }}=0.001\right)$, and reach
\end{abstract}

Patricia M. Livingston

trish.livingston@deakin.edu.au

1 Faculty of Health, Deakin University, Locked Bag 20001, Geelong, Victoria 3220, Australia

2 Institute for Physical Activity and Nutrition Research, School of Exercise and Nutrition Sciences, Faculty of Health, Deakin University, Geelong, Australia

3 Institute of Sport, Exercise and Active Living, College of Sport and Exercise Science, Victoria University, Melbourne, Australia

4 Biostatistics Unit, Faculty of Health, Deakin University, Geelong, Australia distance $\left(M_{\text {diff }}=4.8 \mathrm{~cm}, p_{\text {adj }}=0.024\right)$. A significant difference (unadjusted for multiplicity) in favour of men in the intervention was also found for resting heart rate $\left(M_{\text {diff }}=-3.76\right.$ beats $\min , p=0.034)$. ADT did not modify responses to exercise training.

Conclusions Men with prostate cancer who act upon clinician referrals to community-based exercise training programs can improve their strength, physical functioning, and, potentially, cardiovascular health, irrespective of whether or not they are treated with ADT.

Implications for Cancer Survivors Clinicians should inform men with prostate cancer about the benefits of exercise and refer them to appropriately qualified exercise practitioners and suitable community-based programs.

Trial registration Australia and New Zealand Clinical Trials Register (ANZCTR): ACTRN12610000609055

Keywords Prostate cancer - Androgen deprivation therapy · Aerobic exercise training $\cdot$ Resistance exercise training · Fitness $\cdot$ Physical function

\section{Introduction}

Prostate cancer is the most frequently diagnosed cancer among men in more developed regions of the world [1], with the trend in 5-year relative survival rates approaching $100 \%$ in some counties [2-4]. Men with prostate cancer typically experience adverse effects associated with the disease and its treatment, however, including urinary leakage, bowel urgency, erectile dysfunction [5], fatigue [6], anxiety and depression [7], and diminished health-related quality of life [8]. In addition, those treated with androgen deprivation therapy $(\mathrm{ADT})$ are at increased risk of bone fracture, muscle mass and strength loss, diabetes, obesity, and cardiovascular- 
related mortality $[9,10]$. With this profile of adverse effects, counselling men with prostate cancer to engage in exercise training, in combination with other health promoting activities, has been recommended [11].

Exercise training can improve the health and wellbeing of men with prostate cancer, including aerobic fitness, muscular performance (endurance and strength), fatigue, flexibility, body composition (lean body mass), blood lipids, and quality of life [12-14]. The evidence is less clear, however, on the effects of exercise training on other parameters, such as blood pressure [15-17]. In addition, greater clarity is needed with regard to whether responses to exercise training differ between men with prostate cancer receiving ADT and those not receiving this treatment. Studies conducted thus far have shown minimal differences between these two cohorts $[18,19]$. Given the potential of exercise training to reduce the severity of many of the adverse effects of ADT [10], identifying differences between these two cohorts in their responses to exercise training would inform the design of more effective programs.

One of the features of previous studies involving exercise training and prostate cancer is that trials have been conducted using supervised (i.e. in the presence of a therapist or exercise physiologist) or unsupervised (e.g. homebased without a therapist or exercise physiologist) programs $[13,14]$. Demonstrating the effectiveness of a combined program has value, as men with prostate cancer are likely to have opportunities to undertake supervised and unsupervised exercise training in their communities (e.g. gyms that offer supervised classes and unstructured, selfinitiated training). There seems to be merit in continuing this line of enquiry through designing interventions with features that replicate circumstances that men with prostate cancer are likely to experience.

With exercise training emerging as a key intervention for improving the lives of men with prostate cancer [20], we sought to clarify its effects further in community settings. We report findings from a cluster randomised controlled trial of exercise training for men with prostate cancer. This trial had several features that enhanced the ecological validity of the intervention: (a) clinicians referred men with prostate cancer to the exercise training program, (b) the program involved both supervised training and unsupervised home-based sessions, and (c) the supervised sessions were conducted in community gyms. The primary aim of the study was to investigate the effects of the 12-week exercise training program on fitness and physical function, anthropometric measurements, resting heart rate, and blood pressure. The secondary aim was to determine whether the effects of the program, in terms of exercise outcomes, were different for men who received and those who had not received ADT.

\section{Method}

\section{Design}

This study represents planned secondary analysis of data from a multicentre, cluster randomised controlled trial to determine the efficacy of a clinician referral and 12-week exercise training program for improving exercise levels and quality of life for men with prostate cancer. A detailed description of the study methods is available from the published protocol [21] and the main outcomes have been published [22]. In brief, 15 clinicians were randomised to intervention and control conditions. During consultations, clinicians in both conditions determined each patient's eligibility to participate in the trial. Using short pre-prepared scripts, clinicians recommended eligible patients participate in the trial and advised ineligible patients not to participate [21]. Clinicians in the intervention condition $(n=8)$ referred eligible patients to an exercise training program involving two supervised sessions in community gyms and one unsupervised home-based session per week. Clinicians referred patients using a standardised referral slip, which reiterated the recommendation to participate in the exercise training program. Clinicians in the control condition $(n=7)$ recommended eligible patients participate in the trial and provided usual care regarding advice on exercise training (typically, limited amounts of information are provided). Clinician adherence to the referral process was monitored weekly with emails and personal contact with clinicians at the clinics, checking that they were following the protocol and answering any queries that they may have had. The patients in both conditions completed measures associated with the primary outcomes of the main study [22], and were also invited to take part in measurements of their fitness and physical function, anthropometrics, resting heart rate, and blood pressure. Assessments were conducted before the exercise training program begun (baseline) and following the program (12 weeks).

The protocol for this study received approval from the human research ethics committee of the host university and those of the healthcare services involved with participant recruitment. Each participant provided written informed consent.

\section{Participants}

The 15 clinicians involved in this study practised at urology and radiation oncology outpatient clinics across three major public health services and four private clinics in Melbourne, Australia. Their patients $(n=741)$ were screened to determine their eligibility for this study (see Table 1).

Sample size calculations were based on the primary outcome of the main study (self-reported involvement in moderate and strenuous physical activity) [21, 22], rather than the secondary analysis reported here. 
Table 1 Study inclusion and exclusion criteria

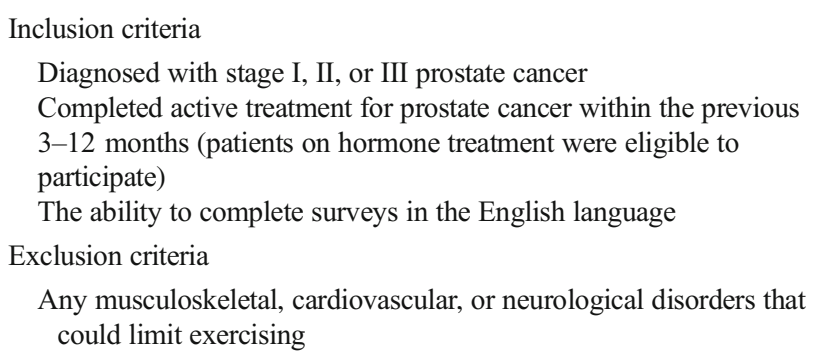

\section{Exercise training intervention}

The 12-week exercise training program involved each participant undertaking two supervised, 50-min sessions at their local community gym and one other home-based session per week. Under the supervision of accredited exercise physiologists (tertiary-educated exercise professionals), postgraduate exercise physiology students from two universities (in training to become accredited exercise physiologists) supervised participants during their gym sessions and provided advice and written instruction for their home-based sessions. The exercise training programs were based on exercise training guidelines for cancer survivors from the American College of Sports Medicine [23] and Exercise and Sport Science Australia [24].

The supervising accredited exercise physiologists have extensive training and experience working with clinical populations, including those with cardiovascular disease, diabetes, cancer, arthritis, and chronic kidney disease. The Masterslevel students, as part of their regular coursework, received education on exercise prescription for people with cancer, as well as on working with various comorbidities that may be present in this clinical population. Prior to training participants in this study, students also received an induction from the supervising accredited exercise physiologists, during which prescription guidelines and training expectations for men with prostate cancer were discussed.

Supervised sessions were performed at community gyms (six YMCA centres and one university-based gym) across Melbourne. The YMCA centres and university-based gym represent accessible and affordable community gyms. For convenience, participants trained at the community gyms located closest to them. Each supervised session included 20$30 \mathrm{~min}$ of aerobic training, prescribed at 40-70\% of maximum heart rate, and 4-6 upper and lower body resistance training exercises, prescribed as two sets of 8-12 repetitions. To achieve consistency in exercise prescription, each participant's program included a $90^{\circ}$ leg press, seated chest press, and seated row. The home-based exercise training programs included both aerobic and resistance exercises, with body weight and resistance-band exercises prescribed to increase the range of exercises participants could perform independently at home. Programs were frequently modified and safely progressed to ensure continual fitness gains.

\section{Outcome measures}

Fitness and physical function Participants completed five functional exercise tests that are common and safe for cancer survivors [25]. Aerobic fitness was assessed using the 6-min walk test [26]. Upper and lower body maximal strength was measured using a one-repetition maximum protocol [25] with the $90^{\circ}$ leg press and seated chest press. Lower body muscular endurance was assessed using the 30-s sit-to-stand test [27]. Balance was assessed using the functional reach test [28].

Anthropometrics Height and body mass were measured using a portable stadiometer (220, Seca, Hamburg, Germany) and portable scales (UC-321, A\&D, Tokyo, Japan), respectively. Body mass index (BMI) was then calculated. Girth measurements of the chest, waist, hips, upper arm, and mid-thigh were taken using standard techniques [29].

Resting heart rate and blood pressure Assessment of resting heart rate (Electro N2965, Polar, Kempele, Finland) and blood pressure (Gamma 4.0 sphygmomanometer, Heine, Herrsching, Germany) were obtained manually. Both measures were taken after each participant had been seated for $10 \mathrm{~min}$.

\section{Statistical analyses}

Data were analysed using SAS software, version 9.3 (SAS Institute, Cary, NC). The baseline characteristics of participants in the intervention and control conditions were compared using chi-squared and Fisher's exact tests for categorical variables (relationship status, highest level of education, stage of disease, treatment regime, ADT, and health service type) and linear mixed models (LMM) with intervention as a fixed effect and clinician as a random effect for continuous variables (age, weeks since active treatment, fitness and physical function, anthropometrics, resting heart rate, and blood pressure). All LMM analyses conducted in this study included clinician as a random effect to account for the clustering due to randomisation at the clinician level.

The effect of the intervention on changes in fitness and physical function, anthropometrics, resting heart rate, and blood pressure between baseline and end of follow-up was determined using LMM with clinician as a random effect. For this analysis, missing data at 12 weeks were imputed using the Markov chain Monte Carlo algorithm with 20 imputations [30].

To evaluate effect modification of the intervention due to ADT, LMM were fitted with intervention, ADT, and the interaction term, intervention $\times$ ADT, as fixed effects and clinician as a random effect. 
Due to the effect of multiple comparisons on experimentwise error (multiplicity), adjusted $p$ values were calculated for the 16 tests included in the primary analysis. Both adjusted and unadjusted $p$ values are reported for these tests. Adjusted $p$ values were computed using Hommel's [31] procedure, which has been shown to have superior statistical power to the original Bonferroni procedure and similar power to other alternatives [32].

\section{Results}

\section{Baseline participant characteristics, attrition, compliance with the intervention, and safety}

Of those screened, 147 patients were eligible and agreed to participate in the main study, with 54 referred from clinicians randomised to the intervention condition and 93 referred from clinicians in the control condition [22]. Of these 147 men, 119 participants (intervention $n=53$, control $n=66$ ) provided data for this secondary analysis. One man withdrew from the intervention condition before baseline data were collected for this secondary analysis, and 27 men from the control condition gave their consent to participate in the main study on the proviso that they did not have to provide physiological data for this secondary analysis. Compared to men who did not provide physiological data, those who did participate in data collection for this secondary analysis were 4.2 years older $(p=0.036)$ and had undergone different treatment regimens (more likely to have had surgery and radiotherapy [24.2 vs $0.0 \%$ ] or radiotherapy and ADT [12.1 vs $0.0 \%$ ], and less likely to have had surgery only [43.9 vs $63.0 \%$ ], radiotherapy only [16.7 vs $18.5 \%$ ], surgery, radiotherapy, and ADT [3.0 vs $11.1 \%$ ], or surgery and ADT [0.0 vs $7.4 \%$ ], $p=0.0006$ ). In addition, 4.5 weeks more had elapsed since active treatment for the men who participated in the data collection $(p=0.032)$.

No significant differences between the intervention and control conditions were observed for any participant characteristic (see Table 2). Of the 119 participants, 11 men (intervention $n=6$, control $n=5$ ) withdrew or were lost to followup (refer to main study paper for details [22]). In comparison to those who underwent assessment at 12 weeks, those who did not attend this assessment had larger chests and waists, as well as higher diastolic blood pressure at baseline $(p<0.05$ for all). No other statistically significant differences were found. Comparisons of the non-attendees from the intervention $(n=6)$ and control $(n=5)$ conditions yielded no statistically significant differences (the power to detect such differences was low, however).

At baseline, compared with men not receiving ADT, those receiving ADT were older $\left(M_{\text {diff }}=7.3\right.$ years, $95 \%$ CI [3.4, 11.2], $p<0.0001)$, had higher systolic blood pressure
$\left(M_{\text {diff }}=8.4 \mathrm{mmHg}, 95 \%\right.$ CI $\left.[1.6,15.2], p=0.016\right)$, walked less distance over $6 \mathrm{~min}\left(M_{\mathrm{diff}}=-44.5 \mathrm{~m}, 95 \%\right.$ CI $[-83.8$, $-5.2], p=0.027)$, and had lower chest strength $\left(M_{\text {diff }}=-13.6 \mathrm{~kg}, 95 \% \mathrm{CI}[-25.2,-2.0], p=0.022\right)$ and leg strength $\left(M_{\text {diff }}=-43.4 \mathrm{~kg}, 95 \%\right.$ CI $[-68.1,-18.8]$, $p=0.0007)$. In addition, for men receiving ADT, more time had elapsed since active treatment $\left(M_{\text {diff }}=5.9\right.$ weeks, $95 \% \mathrm{CI}$ $[0.5,11.3], p=0.034)$.

As has been reported previously, $85 \%$ of the men in the intervention condition undertook at least 18 of the 24 supervised sessions in their local community gyms and, of those who completed their exercise diaries $(74 \%), 81 \%$ completed at least 9 of 12 home-based sessions [22].

Adverse events (musculoskeletal injuries) were reported for two participants. One man in the intervention condition aggravated a previous rotator cuff injury (left shoulder, grade I strain) during exercise training. The other man, in the control condition, aggravated a previous meniscus injury (right knee, inflammation) during baseline testing.

\section{Fitness and physical function}

Sizable, statistically significant differences between conditions were observed, in favour of men in the intervention condition for all measures of physical functioning (Table 3).

Examinations of the possible modifying effect of ADT treatment on the intervention effect revealed minimal, nonsignificant differences between men receiving ADT and those not receiving this treatment. The mean intervention effects were similar for 6-min walk distance (ADT $59.7 \mathrm{~m}, 95 \% \mathrm{CI}$ [11.8, 107.5]; non-ADT 47.5 m, $95 \%$ CI $[21.8,73.2]$; $p=0.673$ ), leg strength (ADT $13.7 \mathrm{~kg}, 95 \%$ CI $[-8.4,35.8]$; non-ADT $25.2 \mathrm{~kg}, 95 \%$ CI $[13.9,36.5] ; p=0.370)$, chest strength (ADT $8.5 \mathrm{~kg}, 95 \%$ CI $[-0.7,17.7]$; non-ADT $7.1 \mathrm{~kg}, 95 \%$ CI $[2.7,11.4] ; p=0.788), 30$-s sit-to-stand result (ADT 4.0 reps, $95 \%$ CI $[0.4,7.6]$; non-ADT 3.4 reps, $95 \%$ CI $[1.5,5.2] ; p=0.778$ ), and reach distance (ADT $7.1 \mathrm{~cm}, 95 \%$ CI $[-0.1,14.4]$; non-ADT $4.1 \mathrm{~cm}, 95 \%$ CI $[0.5,7.7]$; $p=0.487)$.

\section{Anthropometrics}

Differences between conditions were minimal and none were statistically significant (Table 3). Changes over time were also minimal, with the only statistically significant result (unadjusted $p$ only) being a $1.65-\mathrm{cm}$ mean average reduction in hip circumference in favour of the men in the intervention condition.

\section{Resting heart rate and blood pressure}

The resting heart rates of the men in the intervention condition decreased by 3.76 beats/min more than those of men in the control condition (see Table 3). 
Table 2 Baseline participant characteristics

\begin{tabular}{|c|c|c|}
\hline Characteristics & $\begin{array}{l}\text { Intervention }(n=53) \\
\text { Mean } \pm \text { SD } \\
\text { or } n(\%)\end{array}$ & $\begin{array}{l}\text { Control }(n=66) \\
\text { Mean } \pm \text { SD } \\
\text { or } n(\%)\end{array}$ \\
\hline
\end{tabular}

\section{Demographic characteristics}

Age (years)
Relationship status

Married

De facto or living with partner

Separated, divorced, widowed, or not living with partner

Highest level of education

Primary school

Secondary school

Certificate or diploma

University degree

Clinical characteristics

Stage of disease

Stage I

Stage II

Stage III

Treatment regime

Surgery only

Radiotherapy only

Surgery and radiotherapy

Radiotherapy and androgen deprivation therapy

Surgery, radiotherapy, and androgen deprivation therapy

Androgen deprivation therapy

No

Yes

Weeks since active treatment

Health service type

Public

Private

Physical function

6-min walk distance (m)

Leg - 1 repetition maximum $(\mathrm{kg})$

Chest-1 repetition maximum $(\mathrm{kg})$

30-s sit-to-stand (n)

Reach distance $(\mathrm{cm})$

Anthropometrics

Body mass index $\left(\mathrm{kg} / \mathrm{m}^{2}\right)$

Chest circumference $(\mathrm{cm})$

Waist circumference $(\mathrm{cm})$

Hip circumference (cm)

Right thigh circumference $(\mathrm{cm})$

Left thigh circumference $(\mathrm{cm})$

Right arm circumference $(\mathrm{cm})$

Left arm circumference ( $\mathrm{cm}$ )

Resting heart rate and blood pressure

Resting heart rate (beats/min)
$66.8 \pm 8.3$

$41(78.8)$

$3(5.8)$

8 (15.4)

$66.0 \pm 8.1$

0.276

$47(73.4)$

$6(9.4)$

$11(17.2)$

$3(5.8)$

$15(28.8)$

18 (34.6)

$16(30.8)$

19 (46.3)

$22(36.7)$

$11(26.8)$

$29(48.3)$

9 (15.0)

18 (34.0)

0.347

$5(9.4)$

29 (43.9)

15 (28.3)

11 (16.7)

16 (24.2)

12 (22.6)

8 (12.1)

$3(5.7)$

$2(3.0)$

38 (71.7)

$56(84.8)$

10 (15.2)

$5(28.3)$

$26.5 \pm 9.4$

0.586

39 (73.6)

50 (75.8)

16 (24.2)

14 (26.4)

$551.7 \pm 67.9$

0.793

$543.7 \pm 81.8$

$136.8 \pm 42.7$

0.181

$60.5 \pm 22.8$

$60.0 \pm 21.6$

0.540

$14.2 \pm 3.6$

$14.8 \pm 4.1$

0.395

$36.4 \pm 5.8$

$38.2 \pm 7.8$

0.331

$28.5 \pm 3.7$

$28.9 \pm 4.0$

0.510

$101.9 \pm 7.7$

$102.4 \pm 8.3$

1.000

$97.7 \pm 9.9$

$98.3 \pm 10.6$

0.693

$102.2 \pm 6.5$

$103.1 \pm 7.7$

0.671

$47.9 \pm 4.4$

$49.0 \pm 4.7$

0.365

$47.9 \pm 4.2$

$48.6 \pm 4.5$

0.490

$30.5 \pm 2.9$

$30.8 \pm 3.1$

0.723

$30.1 \pm 3.2$

$30.5 \pm 3.1$

0.583

$71.2 \pm 10.9$

$72.0 \pm 13.3$

0.444 
Table 2 (continued)

\begin{tabular}{llll}
\hline Characteristics & $\begin{array}{l}\text { Intervention }(n=53) \\
\text { Mean } \pm \text { SD } \\
\text { or } n(\%)\end{array}$ & $\begin{array}{l}\text { Control }(n=66) \\
\text { Mean } \pm \text { SD } \\
\text { or } n(\%)\end{array}$ & $p^{\text {a }}$ \\
\hline Systolic blood pressure (mmHg) & $\begin{array}{l}134.6 \pm 11.5 \\
83.1 \pm 8.5\end{array}$ & $\begin{array}{l}136.6(11.9) \\
84.5 \pm 8.2\end{array}$ & 0.942 \\
Diastolic blood pressure (mmHg) & 0.448 \\
\hline
\end{tabular}

${ }^{\text {a }}$ Fisher's exact test for categorical variables, linear mixed model including intervention as fixed effect and clinician as random effect for continuous variables. No adjustments were made for multiplicity

\section{Discussion}

This trial demonstrates that the fitness and physical functioning of men with prostate cancer can be enhanced through an exercise training program with features that many men could encounter during their clinical care and within their communities. The intervention commenced with the referral of men with prostate cancer to an exercise training program - an initiative that is consistent with guidelines recommending clinicians counsel prostate cancer survivors to engage in at least 150 min of physical activity per week [11]. At present, no direct formalised pathway exists between oncology clinicians and allied health providers in Australia. People with chronic conditions and complex care needs (including people with cancer), however, may each be eligible for Medicare rebates for up to five allied health consultations per calendar year (including with accredited exercise physiologists [33]) through referrals from general practitioners. Enabling direct referrals between oncology clinicians and allied health professionals, such as exercise physiologists, would facilitate earlier intervention and improve access to exercise training programs for men with prostate cancer. The combination of supervised and unsupervised sessions also matches the reality that most men will be able to access practical support for their physical activity participation from their local communities. Community gyms (and many commercial ones), for example, typically assist members through writing exercise programs for them, providing periodic program reviews, having staff available to demonstrate correct exercise techniques, and running group exercise classes. In addition, YMCA Australia

Table 3 Changes in fitness and physical function, anthropometrics, resting heart rate, and blood pressure, and from baseline to 12 weeks: comparison between conditions

\begin{tabular}{|c|c|c|c|c|c|}
\hline \multirow[t]{2}{*}{ Variable } & \multicolumn{2}{|c|}{ Mean change $(95 \% \mathrm{CI})$} & \multicolumn{3}{|c|}{ Mean intervention effect $(95 \% \mathrm{CI})$} \\
\hline & Intervention $(n=53)$ & Control $(n=66)$ & Intervention-control & $p^{\mathrm{a}}$ & $p_{\text {adj }}^{\mathrm{b}}$ \\
\hline \multicolumn{6}{|l|}{ Fitness and physical function } \\
\hline 6-min walk distance (m) & $64.87(49.76,79.99)$ & $14.89(0.47,29.31)$ & $49.98(28.54,71.42)$ & $<0.0001$ & 0.001 \\
\hline Leg-1 repetition maximum (kg) & $28.54(21.54,35.54)$ & $6.72(-0.06,13.49)$ & $21.82(12.15,31.49)$ & $<0.0001$ & 0.001 \\
\hline Chest -1 repetition maximum $(\mathrm{kg})$ & $10.05(7.30,12.80)$ & $3.14(0.59,5.69)$ & $6.91(3.31,10.51)$ & $<0.0001$ & 0.001 \\
\hline 30-s sit-to-stand (reps) & $3.89(2.80,4.99)$ & $0.51(-0.54,1.56)$ & $3.38(1.87,4.89)$ & $<0.0001$ & 0.001 \\
\hline Reach distance $(\mathrm{cm})$ & $3.17(0.92,5.41)$ & $-1.63(-3.71,0.45)$ & $4.80(1.77,7.82)$ & 0.002 & 0.024 \\
\hline \multicolumn{6}{|l|}{ Anthropometrics } \\
\hline Body mass index $\left(\mathrm{kg} / \mathrm{m}^{2}\right)$ & $-0.02(-0.24,0.20)$ & $0.05(-0.15,0.24)$ & $-0.07(-0.36,0.23)$ & 0.654 & 0.788 \\
\hline Chest circumference $(\mathrm{cm})$ & $-0.75(-1.66,0.16)$ & $-0.01(-0.82,0.79)$ & $-0.74(-1.96,0.49)$ & 0.238 & 0.788 \\
\hline Waist circumference $(\mathrm{cm})$ & $-1.06(-2.21,0.10)$ & $0.06(-0.98,1.09)$ & $-1.11(-2.68,0.46)$ & 0.165 & 0.788 \\
\hline Hip circumference (cm) & $-1.40(-2.35,-0.46)$ & $0.25(-0.61,1.11)$ & $-1.65(-2.95,-0.35)$ & 0.013 & 0.143 \\
\hline Right thigh circumference $(\mathrm{cm})$ & $0.55(-0.15,1.25)$ & $0.24(-0.42,0.90)$ & $0.31(-0.65,1.27)$ & 0.526 & 0.728 \\
\hline Left thigh circumference $(\mathrm{cm})$ & $0.66(-0.03,1.34)$ & $0.35(-0.27,0.97)$ & $0.31(-0.64,1.25)$ & 0.523 & 0.788 \\
\hline Right arm circumference $(\mathrm{cm})$ & $-0.39(-0.99,0.21)$ & $-0.28(-0.81,0.25)$ & $-0.11(-0.92,0.70)$ & 0.788 & 0.788 \\
\hline Left arm circumference $(\mathrm{cm})$ & $-0.34(-0.92,0.25)$ & $-0.20(-0.72,0.32)$ & $-0.14(-0.93,0.65)$ & 0.733 & 0.788 \\
\hline \multicolumn{6}{|l|}{ Resting heart rate and blood pressure } \\
\hline Resting heart rate (beats/min) & $-4.21(-6.76,-1.66)$ & $-0.45(-2.80,1.90)$ & $-3.76(-7.23,-0.28)$ & 0.034 & 0.306 \\
\hline Systolic blood pressure (mmHg) & $-2.04(-4.86,0.79)$ & $1.78(-0.71,4.28)$ & $-3.82(-7.65,0.02)$ & 0.051 & 0.459 \\
\hline Diastolic blood pressure (mmHg) & $-1.31(-3.52,0.90)$ & $0.72(-1.25,2.70)$ & $-2.03(-5.00,0.93)$ & 0.179 & 0.788 \\
\hline
\end{tabular}

${ }^{a} p$ values are unadjusted for multiplicity

${ }^{\mathrm{b}} p$ values are adjusted for multiplicity using Hommel's [31] procedure 
recently announced a commitment to employing accredited exercise physiologists in over 30 facilities by the end of 2018. This development will increase the accessibility of quality support and advice for men with prostate cancer who wish to follow recommendations to be physically active.

Participation in the exercise training improved the aerobic capacity of men with prostate cancer. Not only did the men improve the distances walked over $6 \mathrm{~min}$, but they were also observed to have lower resting heart rates at 12 weeks, which is a common adaption of aerobic exercise training [34]. For the 6-min walk distance, the improvements (ADT $59.7 \mathrm{~m}$, non-ADT $47.5 \mathrm{~m}$ ) were in excess of the recommended criterion for meaningful change in older adults $(20 \mathrm{~m})$ and consistent with the criterion for substantial meaningful change $(50 \mathrm{~m})$ [35]. Given that the participants were already quite physically active at baseline [22] and typically did not attend all the scheduled sessions, these results may be particularly impressive. With regard to the baseline scores for the 6-min walk, for example, the men in this study covered more distance than men with prostate cancer in other studies [36, 37].

The men who participated in the exercise training increased their upper and lower body strength. Given that few randomised controlled trials focusing on these outcomes included men not treated with ADT [38], these observations are an important contribution to the literature. Developing and maintaining muscle strength is imperative, because low muscle strength is an independent predictor of impaired mobility [39] and all-cause mortality [40] in older adults. Consistent with current guidelines [11], clinicians should counsel men with prostate cancer to include resistance exercises as part of their training programs.

Encouraging men with prostate cancer to incorporate resistance exercises as part of their training also provides opportunities for their strength to be monitored (e.g. by exercise physiologists or personal trainers), and for this information to be shared with clinicians. Muscle strength is a better predictor of morbidity and all-cause mortality than muscle mass [39, 40], and the assessment of muscular strength is more feasible than common measures of muscle mass (e.g. via dual-energy X-ray absorptiometry). The assessment of muscular strength could be a valuable outcome from a referred exercise training program, because it could be used for risk stratification and further clinical interpretations.

The effect of the intervention on systolic blood pressure was less pronounced and not statistically significant, but is a finding that warrants further investigation. Previous research on men with prostate cancer has generally shown exercise to have no significant effect on systolic blood pressure [15-17, 41]. Control condition contamination, however, has been cited as a possible reason for the non-significant findings in studies of exercise and prostate cancer [41]. The magnitude of the intervention effect $(-3.82 \mathrm{mmHg})$ is slightly higher than reductions achieved for adults without cardiovascular or other diseases who participate in dynamic resistance training $(-1.8 \mathrm{mmHg})$ [42]. One reason for the muted response may be that the resting systolic blood pressure was not high at baseline (intervention $M=134.6, \mathrm{SD}=11.5$, control $M=136.6, \mathrm{SD}=11.9$ ), with some evidence suggesting that, for people aged over 50, systolic blood pressure $<140 \mathrm{mmHg}$ should be considered normal [43]. Although lowering systolic blood pressure when it is already $<140 \mathrm{mmHg}$ may have limited health benefits (compared with reducing systolic blood pressure to $140-160$ or 140 $149 \mathrm{mmHg}$ ) [44], changes of the magnitude observed in the present trial may be clinically meaningful with men who have higher systolic blood pressure. Clinicians and researchers are encouraged to continue investigations on the effect of exercise training on blood pressure in men with prostate cancer.

The detrimental effect of ADT on fitness and physical functioning observed previously $[36,45,46]$ was also evident in the present study. At baseline, men receiving ADT had less strength, walked shorter distances over $6 \mathrm{~min}$, and had higher systolic blood pressure than those not treated with ADT. Recent research has shown that declines in physical function with ADT do not recover over a 36-month period [36], which underscores the importance of using exercise training as a counteractive measure.

Men treated with ADT responded to the exercise training similarly to men who had not been treated with ADT. This finding adds weight to the small amount of previous research showing minimal, and statistically non-significant, differences between men receiving ADT and those not undergoing this treatment in terms of their responses to exercise training [18, 19]. Although some caution is appropriate when interpreting these findings (due to the comparatively low number of men receiving ADT recruited for the present study), it should be noted that the differences were essentially in favour of men receiving ADT.

A limitation of this trial was that men recruited were more physically active than those reported in other studies [47]. Higher physical activity levels at baseline may have attenuated the magnitude of the effects that may have otherwise been achieved through such an intervention with less active men with prostate cancer. If the men had been less active at baseline, clearer effects may have been observable with respect to resting heart rate, systolic blood pressure, and waist circumference. In addition, as has been mentioned, the comparatively small number of men receiving ADT recruited into this study was another limitation. A larger sample of men with ADT in this study would have enabled firmer conclusions to be drawn about the effects of ADT on men's responses to exercise.

Despite these limitations, the trial demonstrated that clinician referral to a community-based exercise training intervention with supervised and unsupervised sessions can have clear benefits for men with prostate cancer, both in terms of their physical function and, possibly, their cardiovascular health. 
The findings also suggest that favourable outcomes were gained irrespective of whether or not men were treated with ADT. These results should encourage clinicians to inform their patients with prostate cancer about the benefits of exercise and to ensure they are able to refer them to appropriately qualified exercise practitioners or other supervised exercise training programs to develop suitable exercise regimens for these men.

Acknowledgments The authors would like to acknowledge their colleagues who contributed to the main study: Jo Salmon, Kerry S. Courneya, Suzanne Broadbent, Mari Botti, Bridie Kent, and the ENGAGE Uro-Oncology Clinicians' Group.

\section{Compliance with ethical standards}

Funding This study was funded by the Australian Research Council (LP100200176) and the Prostate Cancer Foundation of Australia, with inkind support from YMCA Victoria, Eastern Health, Epworth Healthcare, North Eastern Metropolitan Integrated Cancer Service, and Peter MacCallum Cancer Centre.

Conflict of interest The authors declare that they have no conflict of interest.

Ethical approval All procedures performed in studies involving human participants were in accordance with the ethical standards of the institutional and/or national research committee and with the 1964 Helsinki declaration and its later amendments or comparable ethical standards.

Informed consent Informed consent was obtained from all individual participants included in the study.

Open Access This article is distributed under the terms of the Creative Commons Attribution 4.0 International License (http:// creativecommons.org/licenses/by/4.0/), which permits unrestricted use, distribution, and reproduction in any medium, provided you give appropriate credit to the original author(s) and the source, provide a link to the Creative Commons license, and indicate if changes were made.

\section{References}

1. Ferlay J, Soerjomataram I, Dikshit R, Eser S, Mathers C, Rebelo M, et al. Cancer incidence and mortality worldwide: sources, methods and major patterns in GLOBOCAN 2012. Int J Cancer. 2015;136: E359-86. doi:10.1002/ijc.29210.

2. Siegel R, Ma J, Zou Z, Jemal A. Cancer statistics, 2014. Ca Cancer J Clin. 2014;64:9-29. doi:10.3322/caac.21208.

3. Jung K-W, Won Y-J, Kong H-J, Oh C-M, Shin A, Lee J-S. Survival of Korean adult cancer patients by stage at diagnosis, 2006-2010: national cancer registry study. Cancer Res Treat. 2013;45:162-71. doi:10.4143/crt.2013.45.3.162.

4. Australian Institute of Health and Welfare. Cancer in Australia: an overview 2014 (Cancer series No 90. Cat. no. CAN 88). Canberra: AIHW2014.

5. Wilt TJ, MacDonald R, Rutks I, Shamliyan TA, Taylor BC, Kane RL. Systematic review: comparative effectiveness and harms of treatments for clinically localized prostate cancer. Ann Intern
Med. 2008;148:435-48. doi:10.7326/0003-4819-148-6200803180-00209.

6. Langston B, Armes J, Levy A, Tidey E, Ream E. The prevalence and severity of fatigue in men with prostate cancer: a systematic review of the literature. Support Care Cancer. 2013;21:1761-71. doi:10.1007/s00520-013-1751-5.

7. Watts S, Leydon G, Birch B, Prescott P, Lai L, Eardley S, et al. Depression and anxiety in prostate cancer: a systematic review and meta-analysis of prevalence rates. BMJ Open. 2014;4(3):e003901e. doi:10.1136/bmjopen-2013-003901.

8. Singh J, Trabulsi EJ, Gomella LG. The quality-of-life impact of prostate cancer treatments. Curr Urol Rep. 2010;11(3):139-46. doi:10.1007/s11934-010-0103-y.

9. Taylor LG, Canfield SE, Du XL. Review of major adverse effects of androgen-deprivation therapy in men with prostate cancer. Cancer. 2009;115:2388-99. doi:10.1002/cncr.24283.

10. Storer TW, Miciek R, Travison TG. Muscle function, physical performance and body composition changes in men with prostate cancer undergoing androgen deprivation therapy. Asian J Androl. 2012;14:204-21. doi:10.1038/aja.2011.104.

11. Skolarus TA, Wolf AMD, Erb NL, Brooks DD, Rivers BM, Underwood W, et al. American Cancer Society prostate cancer survivorship care guidelines. Ca Cancer J Clin. 2014;64:225-49. doi:10.3322/caac. 21234 .

12. Hasenoehrl T, Keilani M, Sedghi Komanadj T, Mickel M, Margreiter M, Marhold M, et al. The effects of resistance exercise on physical performance and health-related quality of life in prostate cancer patients: a systematic review. Support Care Cancer. 2015;23:2479-97. doi:10.1007/s00520-015-2782-x.

13. Baumann F, Zopf E, Bloch W. Clinical exercise interventions in prostate cancer patients - a systematic review of randomized controlled trials. Support Care Cancer. 2012;20:221-33. doi:10.1007/ s00520-011-1271-0.

14. Gardner JR, Livingston PM, Fraser SF. Effects of exercise on treatment-related adverse effects for patients with prostate cancer receiving androgen-deprivation therapy: a systematic review. J Clin Oncol. 2014;32:335-46. doi:10.1200/jco.2013.49.5523.

15. Culos-Reed SN, Robinson JW, Lau H, Stephenson L, Keats M, Norris S, et al. Physical activity for men receiving androgen deprivation therapy for prostate cancer: benefits from a 16-week intervention. Support Care Cancer. 2010;18:591-9. doi:10.1007/ s00520-009-0694-3.

16. Galvão DA, Spry N, Denham J, Taaffe DR, Cormie P, Joseph D, et al. A multicentre year-long randomised controlled trial of exercise training targeting physical functioning in men with prostate cancer previously treated with androgen suppression and radiation from TROG 03.04 RADAR. Eur Urol. 2014;65:856-64. doi:10.1016/j. eururo.2013.09.041.

17. Bourke L, Gilbert S, Hooper R, Steed LA, Joshi M, Catto JWF, et al. Lifestyle changes for improving disease-specific quality of life in sedentary men on long-term androgen-deprivation therapy for advanced prostate cancer: a randomised controlled trial. Eur Urol. 2014;65:865-72. doi:10.1016/j.eururo.2013.09.040.

18. Hansen PA, Dechet CB, Porucznik CA, LaStayo PC. Comparing eccentric resistance exercise in prostate cancer survivors on and off hormone therapy: a pilot study. PMR. 2009;1:1019-24. doi:10. 1016/j.pmrj.2009.09.016.

19. Alberga AS, Segal RJ, Reid RD, Scott CG, Sigal RJ, Khandwala F, et al. Age and androgen-deprivation therapy on exercise outcomes in men with prostate cancer. Support Care Cancer. 2012;20:971-81. doi:10.1007/s00520-011-1169-x.

20. Bourke L, Boorjian SA, Briganti A, Klotz L, Mucci L, Resnick MJ, et al. Survivorship and improving quality of life in men with prostate cancer. Eur Urol. 2015;68:374-83. doi:10.1016/j.eururo.2015.04.023.

21. Livingston PM, Salmon J, Courneya KS, Gaskin CJ, Craike M, Botti M, et al. Efficacy of a referral and physical activity program 
for survivors of prostate cancer [ENGAGE]: rationale and design for a cluster randomised controlled trial. BMC Cancer. 2011;11: 237. doi:10.1186/1471-2407-11-237.

22. Livingston PM, Craike M, Salmon J, Courneya KS, Gaskin CJ, Fraser SF, et al. Effects of a clinician referral and exercise program for men who have completed active treatment for prostate cancer: a multicentre cluster randomized controlled trial (ENGAGE). Cancer. 2015;121:2646-54. doi:10.1002/cncr.29385.

23. Schmitz KH, Courneya KS, Matthews C, Demark-Wahnefried W, Galvão DA, Pinto BM, et al. American College of Sports Medicine roundtable on exercise guidelines for cancer survivors. Med Sci Sports Exerc. 2010;42:1409-26. doi:10.1249/MSS. 0b013e3181e0c112.

24. Hayes SC, Spence RR, Galvão DA, Newton RU. Australian association for exercise and sport science position stand: optimising cancer outcomes through exercise. J Sci Med Sport. 2009;12: 428-34. doi:10.1016/j.jsams.2009.03.002.

25. American College of Sports Medicine. ACSM's resource manual for guidelines for exercise testing and prescription. 6th ed. Philadelphia: Lippincott Williams \& Wilkins; 2010.

26. American Thoracic Society. ATS statement: guidelines for the sixminute walk test. Am J Respir Crit Care. 2002;166:111-7. doi:10. 1164/ajrccm.166.1.at1102.

27. Jones CJ, Rikli RE, Beam WC. A 30-s chair-stand test as a measure of lower body strength in community-residing older adults. Res Q Exerc Sport. 1999;70:113-9. doi:10.1080/02701367.1999. 10608028

28. Duncan PW, Weiner DK, Chandler J, Studenski S. Functional reach: a new clinical measure of balance. J Gerontol. 1990;45: M192-7. doi:10.1093/geronj/45.6.M192.

29. Marfell-Jones M, Olds T, Steckler A, Carter L. International standards for anthropometric assessment. Potchefstroom, South Africa: The International Society for the Advancement of Kinanthropometry. 2006.

30. Schafer JL. Analysis of incomplete multivariate data. New York: Chapman \& Hall; 1997.

31. Hommel G. A stagewise rejective multiple test procedure based on a modified Bonferroni test. Biometrika. 1988;75:383-6. doi:10. 1093/biomet/75.2.383.

32. Olejnik S, Li J, Supattathum S, Huberty CJ. Multiple testing and statistical power with modified Bonferroni procedures. J Educ Behav Stat. 1997;22:389-406. doi:10.3102/10769986022004389.

33. Coombes JS, Law J, Lancashire B, Fassett RG. "Exercise is medicine": curbing the burden of chronic disease and physical inactivity. Asia Pac J Public Health. 2015;27:NP600-5. doi:10. 1177/1010539513481492.

34. Levy WC, Cerqueira MD, Harp GD, Johannessen K-A, Abrass IB, Schwartz RS, et al. Effect of endurance exercise training on heart rate variability at rest in healthy young and older men. Am J Cardiol. 1998;82:1236-41. doi:10.1016/S0002-9149(98)00611-0.

35. Perera S, Mody SH, Woodman RC, Studenski SA. Meaningful change and responsiveness in common physical performance measures in older adults. J Am Geriatr Soc. 2006;54:743-9. doi:10. 1111/j.1532-5415.2006.00701.x.
36. Alibhai SMH, Breunis H, Timilshina N, Naglie G, Tannock I, Krahn M, et al. Long-term impact of androgen-deprivation therapy on physical function and quality of life. Cancer. 2015;121:2350-7. doi:10.1002/cncr.29355.

37. Joly F, Alibhai SMH, Galica J, Park A, Yi QL, Wagner L, et al. Impact of androgen deprivation therapy on physical and cognitive function, as well as quality of life of patients with nonmetastatic prostate cancer. J Urol. 2006;176:2443-7. doi: 10.1016/j.juro.2006.07.151

38. Norris MK, Bell GJ, North S, Courneya KS. Effects of resistance training frequency on physical functioning and quality of life in prostate cancer survivors: a pilot randomized controlled trial. Prostate Cancer Prostatic Dis. 2015;18:281-7. doi:10. 1038/pcan.2015.28.

39. Visser M, Goodpaster BH, Kritchevsky SB, Newman AB, Nevitt M, Rubin SM, et al. Muscle mass, muscle strength, and muscle fat infiltration as predictors of incident mobility limitations in well-functioning older persons. J Gerontol A Biol. 2005;60:324-33.

40. Newman AB, Kupelian V, Visser M, Simonsick EM, Goodpaster $\mathrm{BH}$, Kritchevsky SB, et al. Strength, but not muscle mass, is associated with mortality in the health, aging and body composition study cohort. J Gerontol A Biol. 2006;61:72-7.

41. Cormie P, Galvão DA, Spry N, Joseph D, Chee R, Taaffe DR, et al. Can supervised exercise prevent treatment toxicity in patients with prostate cancer initiating androgen-deprivation therapy: a randomised controlled trial. BJU Int. 2015;115:256-66. doi:10. 1111/bju.12646.

42. Cornelissen VA, Smart NA. Exercise training for blood pressure: a systematic review and meta-analysis. J Am Heart Assoc. 2013;2, e004473. doi:10.1161/jaha.112.004473.

43. Taylor BC, Wilt TJ, Welch HG. Impact of diastolic and systolic blood pressure on mortality: implications for the definition of "normal". J Gen Intern Med. 2011;26:685-90. doi:10.1007/ s11606-011-1660-6.

44. James PA, Oparil S, Carter BL, Cushman WC, DennisonHimmelfarb C, Handler J, et al. 2014 evidence-based guideline for the management of high blood pressure in adults: report from the panel members appointed to the Eighth Joint National Committee (JNC 8). JAMA. 2014;311:507-20. doi:10.1001/ jama.2013.284427.

45. Basaria S, Lieb J, Tang AM, DeWeese T, Carducci M, Eisenberger $\mathrm{M}$, et al. Long-term effects of androgen deprivation therapy in prostate cancer patients. Clin Endocrinol. 2002;56:779-86. doi:10. 1046/j.1365-2265.2002.01551.x.

46. Clay CA, Perera S, Wagner JM, Miller ME, Nelson JB, Greenspan SL. Physical function in men with prostate cancer on androgen deprivation therapy. Phys Ther. 2007;87:1325-33. doi:10.2522/ ptj.20060302.

47. Lynch BM, Dunstan DW, Winkler E, Healy GN, Eakin E, Owen N. Objectively assessed physical activity, sedentary time and waist circumference among prostate cancer survivors: findings from the National Health and Nutrition Examination Survey (2003-2006). Eur J Cancer Care. 2011;20:514-9. doi:10.1111/j.1365-2354.2010. 01205.x. 